\title{
Tecnorituales del embarazo: cuerpos de mujer en el origen de la genética medica
}

\author{
Technorituals of pregnancy: \\ Women's bodies at the origin of medical genetics
}

MARÍA JESÚS SANTESMASES*

Resumen: El objetivo de este artículo es mostrar la participación de las mujeres y de su descendencia en la construcción de la genética médica. Las mujeres y la infancia han sido el foco de atención de la genética desde que este espacio biomédico apareció en la clínica entre finales de la década de 1950 y principio de la de 1960 . Esto fue así porque la agenda de la fertilidad ha guiado en buena parte los estudios y los razonamientos sobre la herencia de caracteres en la genética médica. Para desarrollar esta idea presentaré una historia de las imágenes de la genética durante ese periodo, narrativas visuales que transitan de los cuerpos humanos a las imágenes de sus cromosomas, para regresar a los cuerpos, e incluir a la genética medica y sus diagnósticos entre los derechos que la cultura de la reproducción han concedido a las mujeres en la era de la prensa fetal gráfica y la ecografía obstétrica.

Palabras clave: citogenética, embarazo, cromosomas, historia, ecografía.

\begin{abstract}
This article aims at showing how women's bodies and those of their children participated in the construction of medical genetics. Genetics has focused in women and children since this biomedical space emerged in the clinic between the late 1950s and early 1960s. It was so because the fertility agenda guided the studies and ways of thinking on traits' heredity in medical genetics. In order to develop this idea I present a history of genetics' images during that period. Visual narratives transited from human bodies to their chromosomes, to be back to bodies, so as to finally include medical genetics and its diagnosis among the rights that the culture of reproduction has provided to women during the era of foetal press and obstetrics ultrasound scanning.
\end{abstract}

Keywords: citogenetics, pregnancy, chromosomes, history, ultrasound scanning.

Los primeros trabajos de la citogenética humana desde finales de la década de 1950 y hasta mediados de la de 1970 aparecen en forma de una serie histórica de imágenes. Esa era de la prensa fetal gráfica se nutrió de las fotos fetales del fotógrafo sueco Lennart Nilsson, publicadas en 1965, las cuales circularon rápido por espacios geográficos, y profesionales

Recibido: 14/07/2017. Aceptado: 04/09/2017.

* Instituto de Filosofía, Consejo Superior de Investigaciones Científicas, Madrid. mariaj.santesmases@cchs.csic. es Agradezco a Esmeralda Broullon, a las organizadoras del I Congreso Nacional Género, Ciencia y Conocimiento de la Universidad de Cádiz y a dos revisiones anónimas sus comentarios y sugerencias a una versión previa de este artículo. Las investigaciones realizadas han contado con una subvención del Ministerio de Economía y Competitividad (FFI2016-76364). 
(Nilsson et al 1965). Ese conjunto de imágenes popularizaban el feto al tiempo que la clínica se centraba en la fertilidad y el retraso mental. La citogenética médica se combinó en la clínica con la pediatría y la obstetricia al poner el foco de atención en los cuerpos embarazados de las mujeres y en su descendencia: niños y niñas y muy poco después, embriones y fetos.

Inspirada en los análisis de Barbara Duden (1993) sobre la historia fetal, se repasan aquí los primeros usos de la ecografía en las prácticas de diagnóstico prenatal situados en los orígenes de la genética médica (antecedentes en Santesmases 2008, 2014, 2017). El protagonismo de los cuerpos de las mujeres, sometidos al proceso de biomedicalización de la salud y el embarazo, se desplazó a los seres por nacer (Clarke et al., 2003). La introducción del diagnóstico de unos pocos desórdenes cromosómicos fetales creó un nuevo escenario en el que las redes expertas y la cultura material, los aparatos y las técnicas estudiaban embriones y fetos en el interior de los cuerpos de mujeres embarazadas. La transparencia ontológica del cuerpo de la madre se combinaba con el protagonismo creciente del feto para el estudio clínico.

El diagnóstico prenatal, de la salud del feto, empezaba a componerse de un conjunto de técnicas e instrumentos cuyos orígenes son los procedimientos de laboratorio puestos a punto desde el periodo de entreguerras hasta la década de 1960 en estudios de cromosomas y manejo de técnicas citológicas, es decir, de cultivos de células para su estudio. La forma que toman los procedimientos de cultivo celular después de la Segunda Guerra Mundial marcan diferencias que son de género, conceptuales y epistémicas.

En ese trayecto, los cromosomas emergieron como locus sub-celulares en los que la biología y la medicina se encontraron en un momento singular del siglo XX, para observar en ellos y calificar y clasificar lo normal y las anomalías - lesiones, daños o simplemente lo inusual (Kevles 1985, Martin y Lynch 2009, Kottler 1974). El contenido y los significados de esos términos sobre normalidad y patología se construían a partir de evidencias visuales, a través de sucesivas miradas y de sus sesgos. La geografía celular exhibía una iconografía hecha de cromosomas que se sumaba a la morfología humana compuesta de cuerpos y rostros, de facciones cuya relación con los cromosomas estaba construyendo la genética como práctica médica diagnóstica.

Como espacio biomédico, la citogenética humana habría cercado a la medicina, según Peter Keating y Alberto Cambrosio (2003). El laboratorio habría rodeado a la práctica médica y la habría condicionado en buena parte, según estos autores, de forma que la biomedicina consistiría en un desarrollo paralelo del estudio de la biología de lo normal y de las lesiones patológicas. El cuerpo humano sería el producto del reconocimiento médico y del biológico. La biología tomaba forma en los cuerpos y hay una parte del pensamiento y la práctica experimental que se dedicó y se dedica todavía a dilucidar cómo se forma cada parte del cuerpo de cada especie - cómo es que el óvulo fecundado sabría cómo crecer. La vida resultó ser un mecanismo reproductivo y con esa carga de capacidad reproductora llegó a definirse.

Mientras tanto, los cuerpos, a su vez, se desmembraron en partes para dar cuenta de su forma y de sus especificidades, de sus singularidades como especies. A lo largo del siglo $\mathrm{XX}$, el cuerpo se reconstruyó en las muestras extraídas de ellos -la sangre y la orina entre aquellas cuya extracción resultó menos invasiva y su estudio más habitual- de forma que la consulta médica no ha podido separarse del laboratorio sinio que, al contrario, ha estrechado su relación con él. 
Hablo de reconocimiento médico porque el estudio de la citogenética humana fue desde sus inicios un ejercicio médico. La clínica fue el lugar, el espacio institucional y cognitivo de un conjunto de prácticas que quedó impreso en las culturas de nuestro tiempo. La experiencia anatómica estaba contenida en la propia práctica biológica de revelar los cromosomas, procedentes de cuerpos dados por supuestos como resultado de la cultura anatómica (Duden, 1991).

\section{Cuerpos y rostros}

Las imágenes de los cuerpos recogidas en dibujos y fotografías se usaron como representaciones de éstos desde los primeros estudios de la herencia humana en la práctica médica en el siglo XVIII, como ha contado Carlos López Beltrán (2005, 2007). Esas imágenes se tomaban como evidencias, saberes y prácticas incorporados al conocimiento movilizado por la herencia biológica humana desde sus manifestaciones más tempranas. Circularon en papel, enviadas y reenviadas por quienes las producían y las incluían en publicaciones. Y, lo que es más importante pero más difícil de explorar, estaba en el imaginario colectivo el conjunto de facciones clasificables como de retraso o con defectos recogidas por los términos que circulaban - "débiles mentales", "idiotas", "retrasados". Esos términos son hoy hirientes y discriminatorios pero pueden recordarse para dar cuenta de la historia de ese imaginario de la herencia de "defectos" y de sus trayectos (Hogan 2016; Lindee 2006). Aunque el término genética acuñado por William Bateson en Cambridge resultó muy popular, antes de eso la herencia biológica se desplegaba en dibujos y en fotografías. La iconografía genética se concentró en el cuerpo identificado por la medicina, en su anatomía, sus siluetas y contornos.

El protagonismo británico en los orígenes de la genética médica ha situado a Lionel Penrose como agente principal en los primeros años de la genética humana. Esto fue así por sus estudios genéticos de poblaciones humanas, herederos de la cultura eugenésica. Los conceptos y métodos eugenésicos - la promoción de la reproducción de los "seres mejor dotados" - permanecen en permanente revisión desde que los propuso su inventor, Francis Galton, entre 1860 y 1870 (Kevles 1985). A falta de figura semejante en la historiografía de otros países, o al menos a falta de una comunidad heredera de saberes y prácticas de antecesores con un reconocimiento comparable a la británica, la bibliografía sobre Penrose permite repasar aquí algunas de sus ideas y de las imágenes incluidas en sus libros más citados (Laxova 1998).

El libro de Penrose sobre la biología del retraso mental, The biology of mental defect (London: Sidwick and Jackson, 1949), circuló mucho, si hacemos caso del número de reseñas publicadas sobre él. Los asilos y las clínicas para quienes se consideraban con enfermedad mental han sido instituciones útiles para estudiar datos recogidos de forma sistemática sobre de rasgos físicos y su herencia, y su relación con el retraso mental estuvo entre los primeros que se consideraron susceptibles de ser hereditarios (sobre los españoles, del Cura González 2011, 2016). Así presentaba Penrose en ese libro sobre las excepciones y los casos raros que había encontrado en sus estudios - estilos propios de la historia natural asimilados en el conocimiento biológico y en el médico. 
Las investigaciones de Penrose comenzaron después de formarse como psiquiatra y médico, cuando en 1931 recibió el encargo de estudiar al colectivo asilado en la Royal Eastern Counties Institution, compuesto por 1.280 pacientes. Conocido como la Colchester Survey, la investigación pretendía estudiar las causas de la "deficiencia mental" como problema social de naturaleza desconocida. El estudio de convirtió en una enorme colección de datos y en una herramienta que Penrose usó para clasificar los llamados "retrasos" cuando el significado del término incluía enfermedades, desórdenes, síndromes y hasta caracteres de muy variada índole. Esta investigación hizo de Penrose uno de los más influyentes estudiosos británicos de la genética de poblaciones humanas. Clasificó a la gente asilada por medio del estudio de sus habilidades, con pruebas ya entonces en uso, y con reconocimientos médicos. Relacionó síntomas físicos -enfermedades y lesiones- con historias familiares y encontró dificultades para dibujar una línea entre gente normal y la considerada "deficiente", entre causas endógenas y exógenas. De entre las numerosas personas asiladas que estudió, encontró 63 típicos "mongólicos”, con aspecto anatómico característico, con signos corporales singulares. Entre ellos, la edad avanzada de la madre al nacimiento resultó un factor relacionado, aunque también encontró algunos casos nacidos de madres jóvenes (Penrose 1938; Penrose 1951; Gaudillière, 2001). Recogió estadísticas a lo largo de la vida de muchos de ellos, lo que le permitió estudiar el desarrollo de esas diversidades funcionales y su carácter hereditario, cuando se dio el caso.

La infancia fue uno de los principales intereses de Penrose, tanto la normal como aquella clasificada como "con defectos". Muchos genetistas británicos se formaron a su lado o pasaron temporadas en su laboratorio (Laxova 1998). La influencia del pensamiento hereditario de Penrose y el desarrollo en el laboratorio y en la clínica del concepto denominado entonces como "retraso mental" se difundieron entre una comunidad genetista que crecía desde los primeros años de la década de 1960 en número y en técnicas disponibles para diagnosticar y clasificar lesiones y salud.

\section{Citogenética y ecografía en Madrid en la década de $\mathbf{1 9 6 0}$}

La historia del número de cromosomas humanos - cada especie parecía desde principios del siglo XX tener un número característico propio-, del cariotipo humano de 46 cromosomas, que se contaron de forma reproducible desde 1956, es una historia rápida que incluye sucesivas identificaciones de anomalías cromosómicas -numero de cromosomas menor o mayor a los 46 que se tomaron como referencia de normalidad- que se difundieron entre 1959 y los primeros años de la década de 1960 (Tjio y Levan 1956; Ford y Hamerton 1956). El número de esas partículas diminutas encontradas en el núcleo de las células a finales del siglo XIX y así llamadas por que se dejaban teñir para distinguirlas de otros componentes nucleares, permitió diagnosticar el síndrome de Turner y el de Klinefelter como anomalías en los cromosomas que determinan el sexo -el par xx y el par xy- en mujeres y hombres, respectivamente, con problemas de fertilidad (Delgado Echeverría 2007; Sanz 2017). Se identificó también, de forma casi simultánea, el número 47 como propio de la mayoría de los niños y niñas que la clínica diagnosticaba como "mongólicos", que comenzaba a denominarse "síndrome de Down" desde 1960, cuando un grupo de médicos británicos llamó la atención sobre los sesgos racistas que acarreaba el nombre que le había dado John Langdon Down en Inglaterra en 1866 (Down 1866; Wright 2011). 
Fue Marthe Gauthier quien en 1959 puso a punto, a petición de Jerome Lejeune y Raymond Turpin, una técnica de cultivo de tejidos que le permitió identificar a cinco niños y cuatro niñas del Hospital des Enfant Malades de París diagnosticados con "mongolismo" como poseedores un cromosoma adicional al considerado normal. Con aquellos 47 cromosomas, el grupo infantil así clasificado proporcionó un número de cromosomas para el síndrome de Down (Lejeune, Gauthier, Turpin 1959; Gauthier 2009). Aquellas formas faciales y sus capacidades intelectuales asociadas se encontraron con un rasgo en el núcleo de las células somáticas que consistía en un cromosoma adicional, al que se asignó la causa de un defecto congénito.

En 1960 se había diseñado el procedimiento de observación de cromosomas en leucocitos en muestras de sangre periférica. Desde entonces pudieron estudiarse los cromosomas extraídos de muestras de sangre, el número de pruebas y de centros que podían realizarlas aumentó rápido porque la extracción de sangre era ya un práctica médica habitual en los consultorios. Durante unos años, este diagnóstico cromosómico, inicialmente en niños y niñas con síndrome de Down o con desórdenes ligados a la asignación de sexo genital y en parejas infértiles, se realizaba para confirmar o desmentir un diagnóstico obtenido por reconocimiento médico, es decir por la observación clínica de los cuerpos.

Uno de los inicios de las técnicas puestas a punto con fines de diagnóstico genético en España fue el laboratorio de citogenética que se creó en la Clínica de la Concepción de la Fundación Jiménez Díaz en Madrid, dirigido por Andres Sánchez Cascos y Emilia Barreiro. Andrés Sánchez Cascos se había formado en el uso de las técnicas de diagnóstico genético en Londres, junto a uno de los pioneros de la genética médica en Gran Bretaña, Paul Polani. A su regreso, organizó con la joven licenciada en Medicina Emilia Barreiro un servicio de diagnóstico citogenético con las técnicas puestas a punto por Joe Hin Tjio y Albert Levan en Suecia en 1956, y las de Hungerford y Nowell sobre su determinación en sangre basadas en métodos citológicos (Tjio y Levan, 1953; Ford y Hammerton 1956; Nowell, Rowley y Knudson 1998, Lindee 2006) ${ }^{1}$.

Sánchez Cascos se había especializado en cardiología e hizo su tesis para el grado de doctor en Medicina sobre "Correlación electrodinámica de las cardiopatías congénitas" en 1960 en la Universidad de Madrid. ${ }^{2}$ Es decir, se había interesado por la genética humana, y su relación con las cardiopatías.

Uno de los primeros desórdenes en estudiarse por técnicas citogenéticas fue el síndrome de Down. Había sido descrito a mediados del siglo XIX por John Langdon Down asociado a "deficiencia metal" y a caracteres anatómicos concretos, y fue en la década de 1950 cuando se estableció la correlación entre esos caracteres anatómicos y lo que él denominó "mongolismo" con la presencia de un cromosoma más del habitual.

En 1964, Sánchez Cascos y Emilia Barreiro describieron veinte casos de síndrome de Down en un estudio citogenético. Las muestras analizadas procedían de pacientes de la Clínica de la Concepción (Fundación Jiménez Díaz) y del Hospital Clínico, del Hospital de

1 Los experimentos por los cuales Tjio y Levan establecieron en 1956 que el número de cromosomas humano era 46 , y no 48 como hasta entonces se creía, fue posible por el uso de técnicas citogenéticas descritas a principios de la década de 1950. Véase Tjio y Levan 1956, y para una reconstrucción histórica del experimento, Martin, 2004.

2 Tesis inédita, Facultad de Medicina, Universidad de Madrid 1960. 
Niño Jesús, del Hospital Militar y del Hospital de la Cruz Roja. El diagnóstico tenía “utilidad en la práctica por la posibilidad de existencia de portadores sanos entre los familiares de los casos con algún tipo de mongolismo" así como "la futura profilaxis de más de un caso" (Sánchez Cascos, Morales y Barreiro 1964: 23). Es decir, como se habían conocido casos de lesiones hereditarias, podía aconsejarse a la mujer, o a las parejas, no tener más descendencia.

Lo que se denomina "profilaxis" solo podía consistir en evitar un embarazo -con el uso de anticonceptivos en ese momento ilegales todavía en varios países, entre ellos España- o en interrumpirlo (Ignaciuk y Ortiz Gómez 2016, Ortiz-Gómez e Ignaciuk 2018). La promoción de la natalidad y la ilegalidad del aborto, ambas política del franquismo, intervinieron de esta forma en la construcción de los intereses y los objetivos de la genética médica en aquel momento en España. Esas normas limitaron el impacto de las nuevas capacidades diagnósticas también de la citogenética y en algunos casos contribuyeron a que los resultados llevaran a abortos en el extranjero (Nash 1996, Scanlon 1986). Pero no fueron los únicos participantes, pues informaciones y publicaciones médicas y científicas sobre novedades diagnósticas intervinieron en el proceso.

Mientras se empezaban a practicar estas nuevas técnicas, se mantenía al mismo tiempo el valor del diagnóstico del síndrome de Down basado en la uniformidad fisionómica. La importancia atribuida al diagnóstico clínico por los pediatras en la década de 1960 en España se mantenía, sin embargo, y lo hizo hasta la actualidad. A este respecto, son expresivas las afirmaciones del pediatra López Linares, de la Fundación Jiménez Díaz, quien afirmó en 1967 que el "mongolismo" podía diagnosticarse "con una sola mirada" y que "el diagnóstico realizado por un clínico experto era superior, en la mayoría de los casos, al estudio citogenético" (López Linares 1967; Santesmases 2008). Lo cual sugiere que había debates entre genetistas y pediatras en ese momento de mediados de la década de 1960, debates que eran entre dos prácticas epistémicas, la del ojo clínico experto y las nuevas experiencias de diagnóstico genético. En ese debate se concedió superioridad ontológica al laboratorio, de forma que los cromosomas hablarían desde entonces de los cuerpos. Los cromosomas proporcionaban explicaciones de la anatomía y los rasgos faciales y corporales, procedentes del dispositivo subcelular preparado para ser observado al microscopio, superioridad que ya Fiona Miller (2006) había asignado a la determinación del sexo en casos dudosos por métodos anteriores a los estudios cromosómicos.

A medida que fueron descritos, se difundieron las técnicas de identificación de anomalías cromosómicas en análisis citogenéticos, que se incorporaron con rapidez al departamento de Citología de la Fundación Jiménez Díaz. Estas anomalías eran en 1967 un número "casi inagotable". Hasta ese año y desde la creación en 1962 del Departamento de Genética de la Fundación Jiménez Díaz se había hecho el cariotipo a 500 personas: a portadoras de enfermedades, desórdenes o anomalías congénitas y a sus familiares (Sánchez Cascos y Barreiro, 1967, 201).

En cuanto a las técnicas, el segundo curso de genética humana impartido por Sánchez Cascos, Barreiro, Carlos San Román y María Jesús Lautre, publicado en 1967, estaba en buena parte dedicado precisamente a ellas. La "más rápida, cómoda y barata", según ese texto, usaba muestras de sangre, y otra era la extracción del líquido amniótico. Sánchez Cascos menciona que la determinación de cromosomas obtenidos del líquido amniótico 
de mujeres embarazadas por punción del abdomen, podía establecer "con exactitud" el sexo, se entendía que del feto; pero añadía que "dicha punción puede terminar en aborto, no siendo por tanto una técnica recomendable". Finalmente, concluye que la principal aplicación de la genética humana era resolver "el angustioso problema del matrimonio que acaba de tener un hijo malformado o mongoloide y que nos pregunta si tendrá más hijos afectos"3. El texto sugiere que se hacían análisis citogenéticos a niños y niñas de poca edad, y que los resultados se relacionaban con la descendencia futura. La genética incluía rasgos de salud y de enfermedad, y rasgos cromosómicos -desórdenes cromosómicos o anomalías-, y los correlacionaba con la conveniencia o no de tener más descendencia.

Durante la década entre 1965 y 1975 se extendieron esas prácticas diagnósticas del síndrome de Down, tras ponerse a punto la amniocentesis, método de extracción del líquido amniótico por punción abdominal en el cuerpo de las mujeres embarazadas. El procedimiento de estudio de las células fetales contenidas en el líquido amniótico estabilizó la tecnología de identificación de cromosomas humanos y dotó a esta de independencia respecto del examen clínico. La representación de los cuerpos en sus cromosomas empezó a hablar por sí misma, en ausencia de un diagnóstico médico previo del cuerpo de la persona de la que procedía la muestra, ya que el cuerpo fetal no podía ser examinado (Santesmases 2014b). Mientras tanto, desde 1968 el desarrollo de técnicas de ultrasonidos produjo la ecografía, que proporcionaba una imagen fetal de aspecto fotográfico borroso, y cuya tecnología se desarrolló en buena parte asociada a las prácticas clínicas propias de la obstetricia.

El cariotipo fetal -los cromosomas fetales, de un ser por nacer- fue el primero de los pasos en un proceso histórico que situó la representación de ese cuerpo por nacer como autónomo, extraño al cuerpo de la madre. Esa autonomía estaba representada en imágenes. Los secretos que encerraban los cuerpos de las mujeres se habían examinado en las anatomías de sus cadáveres, a la búsqueda de los territorios en los que se generaban los nuevos seres (Park 2006). Una vez abierto, el útero agrandado exhibía su elasticidad como continente y su contenido: un embrión que muchas veces sobrevivía a su madre. El embrión en crecimiento dejó de ser muestra del cuerpo de la madre del que se extrajo y terminó por vivir por su cuenta. El cuerpo materno devino contenedor biológico y legal. La ecografía fetal fue una de las tecnologías que consolidó esa autonomía al producirse las imágenes de su cuerpo en formación. Los contornos aparecían retratados, identificados en los primeros registros de ultrasonidos del ecógrafo "en tiempo real", presumían los textos. El ecógrafo permitía observar el movimiento del cuerpo y el latido del corazón embrionario. Y aunque otras técnicas permitían registrar esos gestos, las imágenes del cuerpo por ultrasonidos son el origen de la consolidación posterior del cuerpo por nacer como sujeto autónomo, desprovisto de su sitio en el útero materno.

En 1970 empezaron a realizarse en el servicio de ginecología del Hospital Clínico de Madrid seguimientos del embarazo y diagnósticos de placenta previa con el ecógrafo Vidoson, a achacar las hemorragias a la localización de la placenta previa a partir de la semana vigésima del embarazo (Parache Hernández y Martínez Hernández 1970). Uno de los usos más eficientes del ecógrafo fue el de participar en las amniocentesis. La pun-

3 Sánchez Cascos (1967). 
ción se realizó al menos desde 1972 en el servicio de ginecología del Hospital Clínico de Madrid con el uso de este aparato que permitía encontrar el lugar "correcto" para tal punción, con el fin de no dañar al feto. La extracción de líquido amniótico es, sin embargo, anterior a la observación fetal. Se realizaba en circunstancias extraordinarias para eliminar exceso de líquido y desde la década de 1950 para determinar el sexo fetal en casos de familias con hemofilias. Durante la década de 1960, la amniocentesis empezó a practicarse para estudiar los cromosomas fetales en búsqueda de un exceso de cromosomas que pudieran asignarse al síndrome de Down. El riesgo de pérdida del embarazo que la extracción misma podía provocar fue disminuyendo a medida que aumentaba la destreza de la extracción (Gaudillière 2001).

En 1973 Parache y María Jesús Lautre mostraron la utilidad del Vidoson en la amniocentesis. Si el palpado contribuyó a ubicar el pinchazo sin perjudicar al feto, y la monitorización proporcionaba el sonido del corazón fetal, que latía más rápido que el de la madre, la ecografía ofrecía certezas visuales (Parache et al. 1973). El conjunto de tecnorituales del embarazo incluía la superioridad ontológica de la instrumentación técnica y en general de los aparatos que desvelaban el interior de los cuerpos, respecto del examen clínico. Cromosomas y nuevos instrumentos se asentaban más poderosos que la observación clínica, en un proceso que ya se había producido en la asignación del sexo "verdadero" en función de la presencia o ausencia de la cromatina de Barr (Ha 2011, Miller 2006, Morgan 2004).

Un vez observada la presencia de células fetales en el liquido amniótico, las técnicas para cultivarlas y observar sus cromosomas fue paulatinamente mejorada con el fin de poder estudiarlas en las primeras semanas del embarazo. En 1975, María Jesús Lautre, que se había formado en la Unidad de Genética de la Fundación Jiménez Diaz con Sánchez Cascos y en el Guy's Hospital de Londres, presentó un conjunto de resultados de estas prácticas. Describió la amniocentesis como una práctica de extracción "por vía transabdominal bajo control ecográfico", lo que minimizaba el riesgo de daños al feto y a la madre. Consistía en la extracción de diez centímetros cúbicos, que se cultivaban después con suero fetal hasta obtener células adecuadas para el estudio de sus cromosomas. La duración del cultivo hasta obtenerlas variaba, pero la premura para obtener resultados no era importante en España, pues el aborto terapéutico no estaba autorizado por la ley, añadía Lautre. De los 67 casos presentados en aquella primera ocasión, dos presentaron alteración cromosómica. Se estaba haciendo posible "el control genético del feto", según Lautre. La prevención "total”, que consistiría en realizar análisis del líquido amniótico a todas las embarazadas, parecía "imposible", escribió, aunque sugirió que podían establecerse algunos criterios en función de riesgos ya descritos. La edad de la madre y la historia familiar eran los principales. "Es misión del médico, concretamente del genetista, informar del riesgo existente”, concluyó (Lautre 1976).

\section{Comentarios finales: imágenes clínicas y prensa fetal gráfica}

El Parlamento aprobó la ley de interrupción del embarazo en junio de 1985, que fue promulgada el 5 de julio. El 31 de julio se publicó una "orden sobre la práctica del aborto en centros o establecimientos sanitarios". En septiembre, un estudio del Instituto de la Mujer sugería que ese bajo número se debía a que las mujeres seguían abortando en la clandes- 
tinidad. Finalmente, el ministerio de Sanidad modificó la norma, de forma que los abortos empezaron a practicarse en centros privados en número creciente mientras en los centros de la red sanitaria pública los servicios alegaban objeción de conciencia, también regulada por la norma, para no realizarlos (Barreiro 2000).

Los procedimientos de la citogenética centraron la atención en los embriones y en los fetos mientras el concepto mismo de embarazo se genetizó, tras medicalizarse (Akrich y Pasveer 1996). La ecografía se sumaban a este conjunto de circunstancias que forman parte de la historia social de las mujeres y del embarazo. La creciente claridad de las fotografías de cromosomas obtenidas por amniocentesis consolidó la autonomía que la cultura y la política contemporáneas adjudicaron al feto. La ecografía, por su parte, no era un retrato químico, sino ultrasónico cuya complejidad técnica incluía desde sus inicios la conversión en imágenes fetales de las ondas de ultrasonidos emitidas y detectadas por el propio instrumento.

Las imágenes fetales públicas, sin embargo, son anteriores a las obtenidas por ecografía. En 1965 la portada de la revista estadounidense Life reproducía una fotografía de un feto suspendido en el espacio. Tomadas por el fotógrafo sueco Lennart Nilsson a embriones obtenidos en cirugías practicadas por razones médicas, fueron publicadas en 1966 por Gaceta Ilustrada en España y por Paris Match en Francia.

La iconografía fetal, por lo tanto, disponía de un conjunto de retratos fotográficos fetales que se constituyó como referente poderoso, y tomaba su forma en esa ampliación al microscopio en color realizada por Nilsson (1965). La observación en vivo, en directo, del movimiento fetal es, por su parte, una representación obtenida por ultrasonidos -con la base tecnológica propia del rádar- que se desarrolló de forma casi simultánea en varios espacios clínicos de Alemania y Gran Bretaña, ligada al diagnóstico prenatal y a la obstetricia, y luego también a otras especialidades médicas. Este conjunto de procedimientos del diagnóstico prenatal cobró con la ecografía un protagonismo intenso, mientras la claridad de las fotografías de Nilsson se mantuvo como referencia.

Entre las prácticas de recolección de formas anatómicas y rasgos físicos asociados a la diversidad funcional y las imágenes fetales en movimiento se extiende el paisaje temporal de construcción de la genética médica contemporánea, que se articuló en torno a la descendencia, la fertilidad y el embarazo. El cuerpo de las mujeres se instalaba así en el primer plano de la agenda biomédica durante la segunda mitad del siglo XX. Se trata de una práctica que discriminaba y regulaba a través de las culturas visuales de una ciencia en imágenes compuesta por cuerpos, cromosomas y árboles genealógicos en el origen de la genética médica.

Las culturas contemporáneas han participado en la facilidad con la que se aceptó la posibilidad de diagnosticar desórdenes en el feto mediante extracción del líquido amniótico. Un resultado positivo de 47 cromosomas, uno más del considerado como correspondiente a un ser sano, puede conducir al denominado aborto terapéutico, a la interrupción voluntaria del embarazo por parte de las mujeres. La autoridad social de la medicina y del laboratorio explicarían, siquiera en parte, la escasa polémica que generaba la interrupción del embarazo en casos de fetos identificados con 47 cromosomas. Junto a ello, una cultura post-eugenésica puedo haber acompañado a la expansión de las culturas de salud pública a lo largo de toda la segunda mitad del siglo XX. La complejidad de la práctica está relacionada también con los derechos de las mujeres a controlar su cuerpo, la anticoncepción y el derecho al aborto. Los médicos conservaron su autoridad al adjudicarse la autorización de anticonceptivos, la 
práctica de la interrupción voluntaria del embarazo y la asistencia a los partos. Ese conjunto de circunstancias configuraba la medicalización creciente no solo del embarazo sino de la cultura científico-médica contemporánea. Esa medicalización incorporó desde sus inicios las imágenes de los cuerpos, la de los cromosomas y las imágenes de embriones y fetos proporcionados por la ecografía y la prensa fetal gráfica desde la década de 1960. Puesto el foco en el ser por nacer, el de las mujeres adquirió una transparencia ontológica creciente mientras participaba en ese proceso.

\section{Referencias}

Akrich, M. y Pasveer, B. (1996): Comment la naissance vient aux femmes: les techniques de l'accouchement en France et aux Pays-Bas, Paris: Synthélabo.

Barreiro, B. (2000): Democracia y conflicto moral: la política del aborto en España e Italia, Madrid: Istmo.

Clarke, A., L. Mamo, J. R. Fosket, J. R. Fishman, and J. K. Shim (2003): "Biomedicalization: Technoscience, Health, and Illness in the US," Annual Review of Sociology 18, pp. 209-32.

del Cura González, M. (2011): Medicina y pedagogía: la construcción de la categoría infancia anormal en España: 1900-1939, Madrid: CSIC.

del Cura, M. (2016): "La subnormalidad a debate: discursos y prácticas sobre la discapacidad intelectual en el segundo franquismo", História, Ciências, Saúde-Manguinhos, 23, pp. 1041-1057.

Delgado Echeverría, I. (2011): El descubrimiento de los cromosomas sexuales, Madrid: CSIC.

Down, J. Langdon H. (1866): "Observation on an ethnic classification of idiots", London Hospital Reports, 3, pp. 259-262.

Duden, B. (1993): Disembodying women: Perspectives on pregnancy and the unborn, Cambridge, Harvard University Press.

Duden, B. (1991): The Woman Beneath the Skin: A Doctor's Patients in Eighteenth-Century Germany, Cambridge, MA: Harvard University Press.

Ford, C., and Hammerton, J. (1956): “The chromosomes of man", Nature 178, pp. 1020-3.

Gaudillière, J.-P. (2001): 'Bettering Babies: Down's Syndrome, Heredity and Public Health in Post-war France and Britain’, en I. Löwy y J. Krige (eds.) Images of Disease. Science, Public Policy and Health in Post-war Europe, Luxemburgo, Office for Official Publications of the European Comunities.

Gauthier, M. (2009): 'Cinquentenaire de la trisomie 21. Retour sur une decouverte', Medicine/Sciences 9 25, 310-316.

Ha, Nathan Q. (2011): “The Riddle of Sex: Biological Theories of Sexual Difference in the Early Twentieth-Century", Journal of the History of Biology 44: 505-546.

Hogan, A. (2016): Life Histories of Genetic Disease, Baltimore: Johns Hopkins University Press.

Ignaciuk, A., y Ortiz Gómez, T. (2016): Anticoncepción, mujeres y género. La “púldora” en España y en Polonia, Madrid, La Catarata.

Jiménez Díaz, C. (1965): La historia de mi instituto, Madrid: Paz Montalvo. 
Keating, P. and Cambrosio, A. (2003): Biomedical platforms: realigning the normal and the pathological in late-twentieth-century medicine, Cambridge, MA, MIT Press.

Kevles, D. J. (1985): In the name of eugenics: Genetics and the uses of human heredity, Cambridge, MA: Harvard University Press.

Kottler, M. J. (1974): "From 48 to 46: Cytological technique, preconception, and the counting of human chromosomes", Bulletin of the History of Medicine, 48, pp. 465.

Lautre, M. J. (1976): "Predicción antenatal de las anomalías cromosómicas", en: J. Botella and L. Izquierdo (eds.), Problemas actuales de genética humana, Madrid: Instituto de España, pp. 177-91.

Laxova, R. (1998): “Lionel Sharples Penrose, 1898-1972: A personal memoir in celebration of the centenary of his birth", Genetics, 150, pp. 1333-1340.

Lejeune, J., Gauthier, M., Turpin, R. (1959): "Study of somatic chromosomes from 9 mongoloid children", Comptes rendus hebdomadaires des seances de l'Academie des sciences, 248, pp. 1721-22.

Lindee, M. S, (2005): Moments of truth in genetic medicine, Baltimore: John Hopkins University Press.

Lippman, A. (1991): "Prenatal Genetic Testing and Screening: Constructing Needs and Reinforcing Inequalities", American Journal of Law and Medicine, 17, pp. 15-50.

López Beltran, C. (2004): El sesgo hereditario: ámbitos históricos del concepto de herencia biológica, Ciudad de México: Universidad Nacional Autónoma de México.

López Beltran, C. (2007): "Hippocratic bodies. Temperament and Castas in Spanish America (1570-1820)", Journal of Spanish Cultural Studies, 8, pp. 253-89.

López Linares, M. (1967): "Síndrome de Down”, en: Sánchez Cascos, A., y Barreiro Miranda, E., Curso de genética humana, Madrid, Fundación Jiménez Díaz, pp. 83-96; en pp. 92-93.

Martin, A. (2004): "Can't any body count? Counting as an epistemic theme in the history of human chromosomes", Social Studies of Science, 34, pp. 923-948.

Martin, A., y Lynch, M. (2009), "Counting things and people: The practices and politics of counting”, Social Problems, 56, pp. 243-266.

Miller, Fiona A. (2006): “'Your true and proper gender': the Barr body as a good enough science of sex", Studies in History and Philosophy of Biological and Biomedical Sciences, 37, pp. 459-483.

Morgan, L. M. (2004): "A social biography of Carnegie embryo no. 836", The New Anatomist, 276, pp. 3-7.

Nash, M. (1996): "Pronatalismo y maternidad en la España franquista", en: G. Bock y P. Thane (eds.), Maternidad y políticas de género, Madrid, Cátedra-Instituto de la Mujer, pp. 279-307

Nilsson, L., A. Ingelman-Sundberg, and C. Wirsén (1965): Ett barn blir till, Stockholm: Bonnier. Versión cast, Un niño va a nacer: el primer drama de la vida. Barcelona: Doyma 1967, trad. cast. J. Masoliver.

Nowell, P., J. Rowley, and A. Knudson (1998): "Cancer Genetics, Cytogenetics - Defining the Enemy Within”, Nature Medicine, 4, pp. 1107-11. 
Ortiz-Gómez, T. e Ignaciuk, A. (2018): The fight for family planning in Spain during late Francoism and the transition to democracy, 1965-1979, Journal of Women's History, (en prensa).

Parache Hernández, J., y Martínez Hernández, H. (1970): “Diagnóstico mediante ecografía bidimensional en obstetricia y ginecología”, Acta Ginecológica XXI: 727-765.

Parache, J., L. Izquierdo, P. Fernández, M. J. Lautre (1973): “Cultivo de líquido amnióticos. Detección de anomalías genéticas”, Acta Ginecológica, 24, pp. 389-396.

Park, K. (2006): Secrets of women: gender, generation, and the origins of human dissection, New York: Zone Books.

Penrose, L. S. (1938): A clinical and genetic study of 1,280 cases of mental defect (Colchester Survey). Special Report Series, Medical Research Council, No 229. Londres: HMSO.

Penrose, Lionel S. (1949): The biology of mental defect, London: Sidwick and Jackson.

Penrose, Lionel S. (1951): "Maternal Age in Familial Mongolism", British Journal of Psychiatry, 97, pp. 738-747.

Sánchez Cáscos, A., Morales, A. y Barreiro, E. (1964): "Estudio genético de veinte casos de síndrome de Down (mongolismo)", Revista Clínica Española, XCIII, pp. 23-26.

Santesmases, M. J. (2008): "Hacia descendencias saludables: algunos orígenes del diagnóstico prenatal en España”, Asclepio, 60, pp. 129-150.

Santesmases, M. J. (2014a): "Superfemale: cromosomas humanos y representaciones del sexo", en: E. Pérez Sedeño y E. Ortega Arjonilla, eds., Cartografías del cuerpo. Biopolíticas de la ciencia y la tecnología, Madrid, Cátedra, pp. 393-422.

Santesmases, M. J. (2014b): "The autonomous karyotype and the origins of prenatal testing: Children, pregnant women and early Down's syndrome cytogenetics, Madrid 19621975", Studies in History and Philosophy of Biological and Biomedical Sciences, 47, pp. 142-153.

Santesmases, M. J. (2017): "Circulating biomedical images: Bodies and chromosomes in the post-eugenic era", History of Science, doi: 0073275317701145.

Sanz, V. (2017): "No Way Out of the Binary: A Critical History of the Scientific Production of Sex", Signs: Journal of Women in Culture and Society 2017, 43, pp. 1-27.

Scanlon, G. (1986): La polémica feminista en la España contemporánea, 1868-1974, Madrid: Akal.

Tijo, Joe H. and Levan, A. (1956): “The chromosome number of man” Hereditas 42, pp. 1-6. Wright, D. (2011): Downs: the history of a disability, Oxford: Oxford University Press. 